\title{
The Effects of Vitamin D on Advanced Glycation End-products in Prediabetic and Type 2 Diabetic Patients with Vitamin D Deficiency
}

\author{
Bahar TEKİN ${ }^{1}$, F. Buket BAYRAM ${ }^{1}$, Dilek GOGAS YAVUZ ${ }^{2}$ \\ ${ }^{1}$ Department of Internal Medicine, Marmara University Hospital, Marmara University School of Medicine, İstanbul, Turkey
}

${ }^{2}$ Department of Internal Medicine, Section of Endocrinology and Metabolism, Marmara University Hospital, Marmara University School of Medicine, İstanbul, Turkey

Aim: There is strong evidence regarding vitamin D being a risk factor for Type 2 Diabetes Mellitus (T2DM). In this study, we aimed to investigate the effects of vitamin D on advanced glycation end-products in prediabetic and type 2 diabetic patients with vitamin D deficiency.

Methods: 40 type 2 diabetic patients, 40 prediabetic patients and 40 healthy controls with the levels of vitamin $D<20 \mathrm{ng} / \mathrm{ml}$ were included in the study. All three groups were given oral vitamin D $50.000 \mathrm{U} / \mathrm{w}$ as loading dose for 8 weeks and it was tapered to maintenance dose to $1500 \mathrm{U} / \mathrm{d}$. All three groups were evaluated with pretreatment and 12th week measurements of height, weight, waist circumference, systolic/diastolic blood pressures, advanced glycation end-products (AGE) in skin, serum CML, fasting blood glucose, HbA1c, 25(OH) vitamin D, calcium, phosphorus, parathyroid hormone(PTH) and their muscle strengths were assessed.

Results: All three groups showed statistically significant increase in their serum $25(\mathrm{OH})$ vitamin D levels $(p<0,0001)$. Serun CML levels increased significantly in all three groups $(p<0,05)$. Skin AGE deposition was observed significantly higher in diabetic group compared to healthy control group $(p<0,05)$; however there was no significant decrease in skin AGE levels in any of the groups after vitamin D replacement $(p>0,05)$. All three groups had statistically significant increase in their muscle strength after treatment $(p<0,0001)$.

\begin{tabular}{|c|c|c|c|c|c|c|c|c|c|}
\hline & \multicolumn{3}{|c|}{ Healty Control } & \multicolumn{3}{|c|}{ Prediabetic } & \multicolumn{3}{|c|}{ Diabetic } \\
\hline & Before & After & $\mathbf{p}$ & Before & After & $\mathbf{p}$ & Before & After & $\mathbf{p}$ \\
\hline $\mathrm{HbA}_{1 \mathrm{C}(\%)}$ & $4,7 \pm 0,34$ & $4,9 \pm 0,4$ & 0,001 & $5,87 \pm 0,3$ & $5,86 \pm 0,4$ & 0,2 & $6,7 \pm 0,7$ & $6,73 \pm 0,9$ & 0,75 \\
\hline FPG (mg/dl) & $87,7 \pm 9,2$ & $87,7 \pm 9$ & 0,98 & $105,2 \pm 14,4$ & $103,4 \pm 13,3$ & 0,19 & $132,5 \pm 31$ & $129,4 \pm 32$ & $\mathbf{0 , 5 6}$ \\
\hline CML (ng/ml) & $504 \pm 285$ & $738 \pm 479$ & 0,001 & $657 \pm 500$ & $903 \pm 571$ & 0,008 & $726 \pm 598$ & $930 \pm 579$ & $\mathbf{0 , 0 0 0 4}$ \\
\hline
\end{tabular}

Discussion: Vitamin D replacement increased serum vitamin D levels in all three groups; however it did not result in significant decrease in glycemic parameters after a 3-month treatment. Serum CML levels significantly increased after treatment and this increase was thought to be due to weight gain. This finding is the first in literature. When AGE deposition in skin is considered a long-term effect, longer periods of follow-up and serum AGE measurements may confirm the correlation. 